NASA Technical Memorandum 100878

\title{
Numerical Analysis of Three-Dimensional Viscous Internal Flows
}

\author{
:NASA-TM-100878) NUMEEICAL ANALYSIS $U F$ \\ THREE-DIMENSIONAL VISCOUS INTERNAL FLOWS \\ (NASA) $10 \mathrm{P} \quad C S C L$ U B \\ N88-21116 \\ Unclas \\ $\mathrm{G} 3 / 010136323$
}

Rodrick V. Chima

NASA Lewis Research Center

Cleveland, Ohio

and

Jeffrey W. Yokota

Sverdrup Technology, Inc.

(Lewis Research Center Group)

NASA Lewis Research Center

Cleveland, Ohio

Prepared for the

First National Fluid Dynamics Congress

cosponsored by the AIAA, ASME, ASCE, SIAM, and APS

Cincinnati, Ohio, July 24-28, 1988

\section{N/SA}




\author{
Rodrick V. Chima \\ NASA Lewis Research Center \\ Cleveland, OH 44135 \\ and \\ Jeffrey W. Yokota \\ Sverdrup Technology, Inc. \\ (Lewis Research Center Group) \\ NASA Lewis Research Center \\ Cleveland, $\mathrm{OH} 44135$
}

\begin{abstract}
A three-dimensional Navier-Stokes code has been developed for analysis of turbomachinery blade rows and other internal flows. The Navier-Stokes equations are written in a Cartesian coordinate system rotating about the $x$-axis, and then mapped to a general body-fitted coordinate system. Streamwise viscous terms are neglected using the thin-layer assumption, and turbulence effects are modelled using the Baldwin-Lomax turbulence model. The equations are discretized using finite differences on stacked C-type grids and are solved using a multistage Runge-Kutta algorithm with a spatially-varying time step and implicit residual smoothing.

Calculations have been made of a horseshoe vortex formed in front of a flat plate with a round leading edge standing in a turbulent endwall boundary layer. Comparisons are made with experimental data taken by Eckerle and Langston for a circular cylinder under similar conditions. Computed and measured results are compared in terms of endwall flow visualization pictures and total pressure loss contours and vector plots on the symmetry plane. Calculated details of the primary vortex show excellent agreement with the experimental data. The calculations also show a small secondary vortex that was not seen experimentally. The calculations required about 1.6 million words of storage and 1.3 hours of CPU time on a Cray X-MP computer.

Calculations have also been made of an annular turbine stator that has been tested experimentally at NASA Lewis. The Mach number ranged from from about 0.21 at the inlet to 0.67 at the exit, and the axial chord Reynolds number was about $1.7 \times 10^{5}$. Computed surface pressure distributions compare well with measured values at three span-wise locations. The endwall boundary layers produce horseshoe vortices at the leading edge of the blade. Computed wake profiles resemble the measured profiles, but computed efficiencies are lower than measured values by a factor of two.
\end{abstract}

\section{INTRODUCTION}

Much of our work in the past few years has involved the analysis of two-dimensional or quasi-threedimensional blade-to-blade flows in turbomachinery ${ }^{1,2}$. In these two references, both Euler and Navier-Stokes results were presented for each blade row considered, and in each case a significant viscous effect was observed. These effects included such things as a pressure-surface or 'cove' separation on an axial turbine blade, a reduction in the choking mass flow and a change in shock location for an axial compressor blade, and a reduction in the peak Mach number and shock strength near the leading edge of a centrifugal impeller. Each of these two-dimensional viscous effects can be expected to lead to secondary flows in three dimensions. Complex geometries, endwall boundary layers, tip clearance effects, etc. also lead to threedimensional flows in turbomachinery. It is the goal of the present work to begin to predict some of these threedimensional viscous effects.

Several steady three-dimensional analyses for turbomachinery have been published lately. Among them are the work of Dawes ${ }^{3}$ and Subramanian and Bozzola ${ }^{4}$. Both used Runge-Kutta schemes implemented on sheared H-type grids. H-grids are particularly easy to generate and implement for turbomachinery calculations, but suffer from poor leading-edge resolution. $\mathrm{Rai}^{5}$ has published a notable analysis of unsteady three-dimensional rotorstator interaction in an axial turbine. He used a thirdorder accurate upwind implicit scheme and a system of patched and overlaid $\mathrm{O}$ - and H-type grids for good resolution of viscous phenomena. His analysis has not been used for steady flows, however.

In this paper we describe a numerical method for analyzing three-dimensional viscous flows in isolated turbomachinery blade passages. The underlying Cartesian formulation allows the method to be applied easily to both Cartesian and cylindrical geometries. Stacked C. type grids give good resolution of critical leading-edge regions.

A multistage Runge-Kutta scheme is used to solve the finite-difference form of the thin-layer Navier-Stokes equations with a Baldwin-Lomax turbulence model. A spatially-varying time step and implicit residual smoothing are used to accelerate convergence of the scheme to a steady state. Two calculations are presented to validate the analysis. The first calculation shows the formation of a horseshoe vortex at the leading edge of a flat plate with a round leading edge that stands in a turbulent endwall boundary layer. Comparisons made between calculated results and experimental data for a circular cylinder under similar conditions show excellent agreement between static pressure distributions and flow visualization pictures on the endwall, and between static and total pressure contours and velocity vector plots made on the symmetry plane. The second calculation is of the flow through an annular turbine stator. Comparisons made between calculated and measured static pressure distributions compare well at three span-wise locations.

\section{GOVERNING EQUATIONS}

The Navier-Stokes equations are written in a Cartesian $(x, y, z)$ coordinate system rotating with angular 
velocity $\Omega$ about the $x$-axis. The rotation introduces source terms in the $y$ - and $z$-momentum equations. The Cartesian equations are mapped to a general body-fitted $(\xi, \eta, \zeta)$ coordinate system using standard techniques. The $\xi$-coordinate direction is assumed to follow the flow direction and the thin-layer approximation is used to drop all viscous derivatives in this direction. All viscous terms in the cross-channel $(\eta, s)$ plane are retained, with an option in the code to delete all cross-derivatives if desired. The resulting equations are as follows.

$\left.\partial_{t} q+J \mid \partial_{\varepsilon} \hat{E}+\partial_{\eta} \hat{F}+\partial_{r} \hat{G}-R e^{-1}\left(\partial_{\eta} \hat{F}_{V}+\partial_{s} \hat{G}_{V}\right)\right]=H$

where:

$\hat{q}=J^{-1}[\rho, \quad \rho u, \quad \rho v, \quad \rho w, \quad e]^{T}$

$H=\quad\left[\begin{array}{llll}0, & 0, & -\Omega_{\rho w}, \quad \Omega \rho v, & 0\end{array}\right]^{T}$

$\hat{E}=J^{-1} \times$

$\left[\rho U^{\prime}, \rho u U^{\prime}+\xi_{x} p, \rho v U^{\prime}+\xi_{y} p, \rho w U^{\prime}+\xi_{x} p, e U^{\prime}+p U\right]^{T}$

$\hat{F}=J^{-1} \times$

$\left.\mid \rho V^{\prime}, \rho u V^{\prime}+\eta_{x} p, \rho v V^{\prime}+\eta_{y} p, \rho w V^{\prime}+\eta_{x} p, e V^{\prime}+p V\right]^{T}$ $\hat{G}=J^{-1} \times$

$\left[\rho W^{\prime}, \rho u W^{\prime}+\zeta_{x} p, \rho u W^{\prime}+\zeta_{y} p, \rho w W^{\prime}+\zeta_{x} p, e W^{\prime}+\left.p W\right|^{T}\right.$

The velocities in $\hat{q}$ are absolute with respect to the coordinate system fixed to the blade. Relative velocities (denoted by a prime) are given by:

$$
\begin{aligned}
u^{\prime} & =u \\
v^{\prime} & =v-\Omega z \\
w^{\prime} & =w+\Omega y
\end{aligned}
$$

and the relative contravariant velocitiy components are given by:

$$
\begin{aligned}
U^{\prime} & =\xi_{x} u+\xi_{y} v^{\prime}+\xi_{z} w^{\prime} \\
V^{\prime} & =\eta_{x} u+\eta_{y} v^{\prime}+\eta_{z} w^{\prime} \\
W^{\prime} & =\zeta_{x} u+\zeta_{y} v^{\prime}+\zeta_{z} w^{\prime}
\end{aligned}
$$

Note that although $u^{\prime}=u, U^{\prime} \neq U$.

The energy and static pressure are given by:

$$
\begin{gathered}
e=\rho\left[C_{v} T+\left(u^{2}+v^{2}+w^{2}\right) / 2\right] \\
p=(\gamma-1)\left[e-\rho\left(u^{2}+v^{2}+w^{2}\right) / 2\right]
\end{gathered}
$$

Using Stoke's hypothesis, $\lambda=-\frac{2}{3} \mu$, the viscous fux $\hat{F}_{V}$ can be written as follows:

$$
\hat{F}_{V}=J^{-1} \mu\left[0, F_{2}, F_{3}, F_{4}, F_{5}\right]^{T}
$$

where

$$
\begin{aligned}
& F_{2}=C_{1} \partial_{\eta} u+C_{2} \eta_{x}+C_{3} \partial_{s} u-C_{4} \eta_{x}+C_{5} \zeta_{x} \\
& F_{3}=C_{1} \partial_{\eta} v+C_{2} \eta_{y}+C_{3} \partial_{s} v-C_{4} \eta_{y}+C_{5} \zeta_{y} \\
& F_{4}=C_{1} \partial_{\eta} w+C_{2} \eta_{x}+C_{3} \partial_{s} w-C_{4} \eta_{z}+C_{5} \zeta_{z} \\
& F_{5}=\frac{\mu \gamma}{P_{r}}\left(C_{1} \partial_{\eta}\left(C_{v} T\right)+C_{3} \partial_{s}\left(C_{v} T\right)\right)+u F_{2}+u F_{3}+w F_{4}
\end{aligned}
$$

and

$$
\begin{aligned}
& C_{1}=\eta_{x}^{2}+\eta_{y}^{2}+\eta_{z}^{2} \\
& C_{2}=\frac{1}{3}\left(\eta_{x} \partial_{\eta} u+\eta_{y} \partial_{\eta} v+\eta_{z} \partial_{\eta} w\right) \\
& C_{3}=\eta_{x} \zeta_{x}+\eta_{y} \zeta_{y}+\eta_{x} \zeta_{z} \\
& C_{4}=\frac{2}{3}\left(\zeta_{x} \partial_{\zeta} u+\zeta_{y} \partial_{s} v+\zeta_{z} \partial_{s} w\right) \\
& C_{5}=\eta_{x} \partial_{s} u+\eta_{y} \partial_{s} v+\eta_{z} \partial_{s} w
\end{aligned}
$$

Terms multiplied by $C_{1}$ and $C_{2}$ lead to non-mixed second derivative viscous terms like $u_{\eta \eta}$, while terms multiplied by $C_{3}-C_{5}$ lead to mixed-derivative terms like $u_{n 5}$. The viscous $f$ ux vector $\hat{G}$ can be written similarly, with directions $\eta$ and $\zeta$ everywhere interchanged.

Metric terms are defined using the following relations.

$$
\begin{aligned}
& {\left[\begin{array}{lll}
\xi_{x} & \eta_{x} & s_{z} \\
\xi_{y} & \eta_{y} & \varsigma_{y} \\
\xi_{z} & \eta_{z} & \varsigma_{z}
\end{array}\right] } \\
&=J\left[\begin{array}{lll}
y_{\eta} z_{\zeta}-y_{\zeta} z_{\eta} & y_{\zeta} z_{\xi}-y_{\xi} z_{\zeta} & y_{\xi} z_{\eta}-y_{\eta} z_{\xi} \\
x_{\zeta} z_{\eta}-x_{\eta} z_{\zeta} & x_{\xi} z_{\zeta}-x_{\zeta} z_{\xi} & x_{\eta} z_{\xi}-x_{\xi} z_{\eta} \\
x_{\eta} y_{\xi}-x_{\zeta} y_{\eta} & x_{\zeta} y_{\xi}-x_{\xi} y_{\zeta} & x_{\xi} y_{\eta}-x_{\eta} y_{\xi}
\end{array}\right]
\end{aligned}
$$

where

$J=$

$\left(x_{\xi} y_{\eta} z_{\xi}+x_{\varsigma} y_{\xi} z_{\eta}+x_{\eta} y_{\varsigma} z_{\xi}-x_{\xi} y_{\varsigma} z_{\eta}-x_{\eta} y_{\xi} z_{\varsigma}-x_{\varsigma} y_{\eta} z_{\xi}\right)^{-1}$

The equations are nondimensionalized by arbitrary reference quantities (here the inlet total density poref and the total sonic velocity $c_{0 r e f}$ were used, and the Reynolds number Re and Prandtl number $\operatorname{Pr}$ are defined in terms of these quantities. The equations assume that the specific heats $C_{p}$ and $C_{v}$ and Prandtl number are constant, that Stoke's hypothesis is valid, and that the effective viscosity for turbulent flows may be written as

$$
\mu_{e f f}=\mu_{\text {am }}+\mu_{t u r b}
$$

where the laminar viscosity is calculated using a power law function of temperature:

$$
\frac{\mu_{\text {lam }}}{\mu_{\text {ref }}}=\left(\frac{T}{T_{\text {ref }}}\right)^{n}
$$

with $n=\frac{2}{3}$ for air.

\section{TURBULENCE MODEL}

The Baldwin-Lomax algebraic two-layer eddy viscosity model ${ }^{6}$ is applied on cross-channel $(\eta, \zeta)$ planes. T wo modifications to the standard model are made to account for the endwall boundary layer and the blade boundary layer and wake, and their interaction in corners.

First, the distance from the wall is calculated using the Buleev ${ }^{7}$ length scale $d$ :

$$
d=\frac{2 s_{\eta} s_{r}}{s_{\eta}+s_{s}+\left(s_{\eta}^{2}+s_{s}^{2}\right)^{1 / 2}}
$$

where $s_{\eta}$ and $s_{s}$ are normal distances from the walls in the $\eta$ - and 5 -directions respectively. This length scale 
has the desirable property that $d$ approaches the normal distance from one wall at large distances from the other wall.

Secondly the turbulent viscosities are calculated across each boundary layer or wake separately, then the total turbulent viscosity is taken as the vector sum of the components, i.e.,

$$
\mu_{t u r b}=\left(\mu_{t u r b \eta}^{2}+\mu_{t u r b s}^{2}\right)^{1 / 2}
$$

This assumption has the desirable properties that outside of one viscous layer $\mu_{\text {turb }}$ takes on values calculated for the other layer, that it goes to zero in the core flow, and that near corners it accounts for both walls.

\section{COMPUTATIONAL GRID}

Two-dimensional body-fitted grids for this work were generated using the GRAPE code developed by Sorenson ${ }^{8}$. Three-dimensional grids were formed by stacking the 2-D grids. Figure 1 shows a 3-D grid around a plate with a round leading edge. For annular geometries the 2-D grids were stacked along a radial stacking line and stretched in the $\eta$-direction so that the blade shape remained constant and the angular pitch of the outer (periodic) boundary remained constant.

\section{BOUNDARY CONDITIONS}

At the inlet, total temperature $T_{0}$ ref is specified as a constant. A $\zeta$-distribution of total pressure $\left(P_{0} / P_{0}\right.$ ref $)$ is specified, $c / 2 / 2$ - as a constant or as appropriate for an inlet boundary layer with given thickness and a powerlaw velocity profile. For Cartesian geometries the $(x, y)$ and $(x, z)$ flow angles are specified. For cylindrical geometries the $(x, y)$ flow angle is replaced by the inlet whirl $r v_{\theta}$.

For supersonic inlet flows, all flow variables are specified at the inlet. For subsonic flows the inlet conditions are updated each iteration by extrapolating the upstream-running Riemann invariant $R^{-}$based on the absolute total velocity $Q=\sqrt{u^{2}+v^{2}+w^{2}}$ to the inlet.

$$
R^{-}=Q-\frac{2 c}{\gamma-1}
$$

The total velocity is then found from the $T_{0}$ rcf using:

$$
Q=\frac{(\gamma-1) R^{-}+\sqrt{2(1-\gamma)\left(R^{-}\right)^{2}+4(\gamma+1) C_{p} T_{0} \text { rcf }}}{(\gamma+1)}
$$

Velocity components are found from $Q$ and the specifed angles or whirl. Within the endwall boundary layer, that is, where $P_{0} / P_{\text {Oref }}<1$, the $\mathrm{v}$ and $w$ velocity components are found by extrapolation from upstream. The density is found using:

$$
\frac{\rho}{\rho_{0 \text { ref }}}=\frac{P_{0}}{P_{0 \text { ref }}}\left[\frac{\gamma-1}{2} \frac{\left(Q-R^{-}\right)}{c_{0 \text { ref }}}\right]^{\frac{\gamma}{\gamma-1}}
$$

For subsonic outflow the exit static pressure is specified and $(\rho, \rho u, \rho v, \rho w)$ are extrapolated. For Cartesian geometries the exit pressure is constant. For annular geometries the hub pressure is specified and the radial pressure distribution is found by integrating the axisymmetric radial momentum equation:

$$
\frac{d p}{d r}=\frac{\rho v_{\theta}^{2}}{r}=\frac{\rho}{r^{3}}(v z-w y)^{2}
$$

Sidewalls and the trailing-edge cut are treated as periodic boundaries.

On the blade surface $V^{\prime}=0$, and for viscous flows $U^{\prime}=W^{\prime}=0$. Blade surface pressures are found from the normal momentum equation. On the hub $(\varsigma=1)$ and tip $\left(s=\zeta_{\max }\right)$ :

$$
\begin{gathered}
\left(\zeta_{x} \xi_{x}+\zeta_{y} \xi_{y}+\zeta_{z} \xi_{z}\right) \partial_{\xi} p+\left(\zeta_{x} \eta_{x}+\zeta_{y} \eta_{y}+\zeta_{z} \eta_{z}\right) \partial_{\eta} p \\
+\left(\zeta_{x}^{2}+\zeta_{y}^{2}+\zeta_{z}^{2}\right) \partial_{\zeta} p=-\rho\left[\Omega\left(\zeta_{y} w-\zeta_{z} v\right)\right. \\
\left.+U^{\prime}\left(\zeta_{x} \partial_{\xi} u+\zeta_{y} \partial_{\xi} v+\zeta_{z} \partial_{\xi} w\right)+V^{\prime}\left(\zeta_{x} \partial_{\eta} u+\zeta_{y} \partial_{\eta} v+\zeta_{z} \partial_{\eta} w\right)\right]
\end{gathered}
$$

On the blades $(\eta=1)$ the normal momentum equation can be found from (18) by replacing $s$ everywhere by $\eta$ and $V^{\prime}$ by $W^{\prime}$.

\section{MULTISTAGE RUNGE-KUTTA ALGORITHM}

The governing equations are discretieed using a node-centered finite difference scheme. Second order central differences are used throughout.

The multistage Runge-Kutta scheme developed by Jameson, Schmidt, and Turkel ${ }^{9}$ is used to advance the flow equations in time from an initial guess to a steady state. If we rewrite (1) as

$$
\left.\partial_{t} q=-J \mid R_{I}-\left(R_{V}+D\right)\right]
$$

where $R_{I}$ is the inviscid residual including the source term, $R_{V}$ is the viscous residual, an $\mathrm{D}$ is an artificial dissipation term described in the next section, then the multistage Runge-Kutta algorithm can be written as follows:

$$
\begin{aligned}
q_{0} & =q_{n} \\
q_{1} & =q_{0}-\alpha_{1} J \Delta t\left[R_{I} q_{0}-\left(R_{V}+D\right) q_{0}\right] \\
& \vdots \\
q_{k} & =q_{0}-\alpha_{k} J \Delta t\left[R_{I} q_{k-1}-\left(R_{V}+D\right) q_{0}\right] \\
q_{n+1} & =q_{k}
\end{aligned}
$$

For efficiency both the physicial and artificial dissipation terms are calculated only at the first stage, then are held constant for subsequent stages.

\section{ARTIFICIAL DISSIPATION}

The dissipative term $D$ in (20) is a nonconservative version of that used by Jameson et al. ${ }^{0}$ It is given by:

$$
D_{q}=\left(D_{\varepsilon}+D_{\eta}+D_{s}\right) q
$$


where the $\xi$-direction operator is given by

$$
D_{\ell q}=C\left(V_{2 q \varepsilon \varepsilon}-V_{1 q \varepsilon \varepsilon \varepsilon \varepsilon}\right)
$$

where

$$
C=\frac{1}{J \Delta t}
$$

is a coefficient that cancells similar terms in (21). To minimize the artificial dissipation in viscous regions we reduce $\mathrm{C}$ linearly across several grid points to zero at the walls.

The terms $V_{2}$ and $V_{1}$ are given by:

$$
\begin{aligned}
& V_{2}=\mu_{2} \max \left(\nu_{i+1}, \nu_{i}, \nu_{i-1}\right) \\
& V_{4}=\max \left(0, \mu_{4}-V_{2}\right)
\end{aligned}
$$

where

$$
\nu_{i, j}=\frac{\left|P_{i+1, j}-2 P_{i, j}+P_{i-1, j}\right|}{\left|P_{i+1, j}+2 P_{i, j}+P_{i-1, j}\right|}
$$

and

$$
\begin{aligned}
& \mu_{2}=O(1) \\
& \mu_{4}=O\left(\frac{1}{16}\right)
\end{aligned}
$$

In smooth regions of the flow the dissipative terms are of third order and do not detract from the formal secondorder accuracy of the scheme. Near shocks $\nu_{i, j}$ is large and the dissipative terms become locally of first order.

\section{THREE-DIMENSIONAL STABILITY LIMIT}

Applying a linear stability analysis to the inviscid form of (20-21) gives the following expression for the time step.

$$
\Delta t \leq \frac{C F L}{l_{x}|u|+l_{y}\left|v^{\prime}\right|+l_{x}\left|w^{\prime}\right|+c \sqrt{l_{x}^{2}+l_{y}^{2}+l_{x}^{2}+\Omega^{2}}}
$$

where

$$
\begin{aligned}
& l_{x}=\left|\xi_{x}\right|+\left|\eta_{x}\right|+\left|s_{x}\right| \\
& l_{y}=\left|\xi_{y}\right|+\left|\eta_{y}\right|+\left|s_{y}\right| \\
& l_{x}=\left|\xi_{x}\right|+\left|\eta_{x}\right|+\left|s_{x}\right|
\end{aligned}
$$

The Courant limit for a particular multistage scheme depends on the number of stages and the choice of coefficients $\alpha_{i}$. See Ref. 9 for several examples.

To accelerate convergence to a steady state we use the maximum permissible time step at each grid point so that the Courant number is constant everywhere. The time step is calculated once based on the initial conditions. It is stored and is not updated during the calculations.

\section{IMPLICIT RESIDUAL SMOOTHING}

Residual smoothing was introduced by Lerat (see for example Ref. 10) for use with the Lax-Wendroff scheme and was later applied to Runge-Kutta schemes by
Jameson $^{21}$. The technique involves replacing the residual calculated in (20) with a value that has been smoothed by an implicit filter, i.e.,

$$
\left(1-\epsilon_{\xi} \delta_{\xi \xi}\right)\left(1-\epsilon_{\eta} \delta_{n \eta}\right)\left(1-\epsilon_{\varsigma} \delta_{\varsigma \zeta}\right) R=R
$$

where $\delta_{E \varepsilon}, \delta_{\eta \eta}$, and $\delta_{\xi \xi}$ are standard second difference operators and $\epsilon_{\xi}, \epsilon_{\eta}$, and $\epsilon_{\xi}$ are smoothing parameters.

Linear stability analysis has shown that the RungeKutta scheme with implicit residual smoothing may be made unconditionally stable if the $\epsilon$ smoothing parameters are made sufficiently large. In one dimension

$$
\epsilon \geq \frac{1}{4}\left[\left(\frac{\lambda}{\lambda^{*}}\right)^{2}-1\right]
$$

gives unconditional stability if $\lambda^{*}$ is the Courant limit of the unsmoothed scheme, and $\lambda$ is a larger operating Courant number. In three dimensions different $\epsilon$ 's may be used in each direction, and their magnitudes may be often be reduced below the value given by $\mathrm{Eq}$. (25.)

\section{RESULTS}

Two sets of computed results are presented for preliminary validation of the code described above. The first set of results shows the structure of a horseshoe vortex formed at the base of a cylinder standing in a turbulent boundary layer. The second set of results is for turbulent flow through an annular turbine cascade. Computed results are compared to experimental data in each case.

When a boundary layer approaches a local obstruction such as the leading edge of a turbine blade, the low momentum fluid in the boundary layer often cannot overcome the local pressure gradient and the flow separates from the wall. In front of the obstacle the separation creates a vortex which convects around the sides of the obstacle and forms a characteristic horseshoe-shaped flow region.

Eckerle and Langston ${ }^{12}$ have made detailed measurements of the horseshoe vortex in front of and around a cylinder of diameter $D$ centered between the sidewalls of a wind tunnel. Test conditions included an inlet Mach number of $0.084, R e_{D}=5.5 \times 10^{5}$, and an upstream turbulent boundary layer thickness $\delta=0.1 D$. Detailed surface flow visualization and static pressure measurements were made, and static and total pressure measurements were taken using a five-hole probe.

Figure 1 shows the grid used to compute Eckerle and Langston's flow. The grid shown has been coarsened for clarity. The actual grid had $65 \times 49 \times 25$ points with an initial spacing at the walls $\Delta s_{i}=0.001 D$. To avoid questions of trailing-edge vortex shedding, a tail board was added from the back half of the cylinder to the exit boundary. The base grid is approximately 6D square and $.5 \mathrm{D}$ high, to match the dimensions of Eckerle and Langston's wind tunnel test section. We used a symmetry condition at mid span but computed the full symmetric flow side-to-side.

The experimental inlet Mach number of 0.084 is too low for the compressible algorithm used here, so the calculations were run with $M_{\text {in }}=0.2$. The peak Mach 
number was 0.34 at the cylinder-plate junction, so the flow was essentially incompressible. The Reynolds number and inlet boundary layer thickness were matched to the experimental data.

Even with $M_{\text {in }}=0.2$ the four-stage Runge-Kutta scheme seemed to converge poorly, so we eventually ran the calculations with a two-stage scheme with $\alpha_{i}=$ $(1.2,1),. C F L=4$., and implicit residual smoothing at each stage with $\epsilon_{\xi}=2$., $\epsilon_{\eta}=\epsilon_{q}=4$. The initial convergence rate was fast, as shown in Fig. 2 by the histories of the maximum and rms residuals, but after 1000 iterations the solution showed only a tiny horseshoe vortex that did not match the data. Over the next 3000 iterations the residuals changed little, but the vortex grew and moved upstream until it stabilized at the position shown later. The total solution took 1.3 hours on a Cray $\mathrm{X}$-MP computer.

Figures 3 and 4 compare the calculated and experimental data on the endwall. Figure 3 shows contours of constant static pressure coefficient, defined by:

$$
C_{p o}=\frac{P_{0}-P_{0} \text { ref }}{P_{0 \text { ref }}-P_{0 \text { ref }}}
$$

Computed contours are on the bottom and measured contours are on the top. The calculations show excellent agreement with the data from the symmetry plane to about 45 degrees around the cylinder, where the influence of the tailboard becomes apparent. The lower part of Figure 4 shows a flow visualization picture made with ink dots on the endwall. It clearly shows the separation line and reverse flow region ahead of the cylinder. The computed vector plot at the top of the figure shows close agreement with the measured separation line location and flow directions. The vectors are one point off the endwall, and are all drawn to the same length, so they show direction only.

Figure 5 compares the static pressure coefficient distributions on the endwall along the symmetry line ahead of the cylinder. The experimental data (circles) show a general pressure rise upstream due to the cylinder blockage, but also a large dip in the pressure inside the separated region. Two vertical bars indicate substantial unsteadiness in the experimental data. Eckerle and Langston included a 2-D potential solution (solid line) for comparison. The computed solution (line with triangles) shows good agreement with the data ahead of the saddle point, but falls short of predicting the magnitude of the pressure dip. The discrepancy may be due to the unsteadiness in the real flow or to lack of resolution in the computed solution.

Figure 6 compares computed (bottom) and experimental (top) velocity vectors and total pressure loss coefficient contours on the symmetry plane upstream of the cylinder. The total pressure loss coefficient is defined by:

$$
C_{p t}=\frac{P_{0 \text { ref }}-P_{0}}{P_{0 \text { ref }}-P_{\text {ref }}}
$$

These contours show nearly horizontal boundary layerlike flow upstream that rolls up into a vortex with a high loss core. The low loss fluid above the boundary layer curves down the face of the cylinder and carries high momentum fluid to the region near the cylinderendwall junction. There is excellent agreement between the computed and experimental data.

The velocity vectors show how the flow rolls up to form a horseshoe ahead of the cylinder. Experimental velocity vectors are missing in areas where the flow angle exceeded the calibrated range of the five-hole probe. From this data Eckerle and Langston ${ }^{12}$ concluded that "The reverse flow did not roll up to form a vortex, however. The vectors clearly show that a closed vortex was not present in the plane of symmetry, though positive pitch angles in a portion of the reverse flow at $R / D=0.72$ and $Y / D=0.02$ may indicate the start of vortex formation. Rather than rolling up, flow passed out of the plane and proceeded tangentially around the cylinder."

The computed vectors clearly show a vortex in the symmetry plane. A small counterrotating secondary vortex is also shown at the cylinder-endwall junction. The dimension of the secondary vortex is about 1.5 times the diameter of the five-hole probe, and would have been nearly impossible to detect experimentally.

The second set of results is for an annular cascade of constant profile turbine stator vanes developed and tested at NASA Lewis ${ }^{13,14}$. The annular ring has 36 vanes with a hub-tip radius ratio of 0.85 and a tip diameter of $508 \mathrm{~mm}$. The vanes themselves are $38.10 \mathrm{~mm}$ high and have an axial chord of $38.23 \mathrm{~mm}$. Design flow conditions are for a fully axial inflow with a hub-static to inlet-total pressure ratio of 0.6705 . These conditions correspond to average inlet and exit Mach numbers of 0.211 and 0.665 respectively. The Reynolds number based on axial chord is $1.73 \times 10^{5}$.

A grid consisting of $97 \times 31 \times 33$ points with an initial spacing at the wall of 0.0002 of a blade chord was used for the flow calculations and is shown in Figure 7.

The calculation was run with a four-stage scheme with $\alpha_{i}=(1 / 4,1 / 3,1 / 2,1)$ and $C F L=5.5$, using implicit residual smoothing after each stage with $\epsilon_{\xi}=$ $\epsilon_{\eta}=\epsilon_{r}=0.75$. Convergence histories for the annular cascade calculation are shown in Fig. 8 where the log of the maximum and rms-averaged residuals have dropped approximately 3.5 orders of magnitude in 1500 iterations. The total CPU time was approximately 54 minutes for this calculation.

Mach number contours at mid span are shown in Fig. 9 to illustrate the blade boundary layer and wake thicknesses. There are approximately 12 grid points across the pressure surface boundary layer.

The inlet boundary later thicknesses were specifed as 1.9 percent span on the hub and 7.1 percent span on the tip, corresponding to the measured data in Ref. 13. In Fig. 10, velocity vectors with superimposed contours of total pressure show how these boundary layers roll up into horseshoe vortices at the leading edge of the blade. The primary vortices are considerably smaller than the inlet boundary layers, and each primary vortex has an even smaller counterrotating secondary vortex associated with it.

Figure 11 shows a comparison between the calculated surface static pressure distribution and data obtained by Goldman and Seasholtz ${ }^{13}$ at locations of 13.3 , 50.0 , and 86.7 percent span. The calculated results agree very well with the experimental data at all spanwise lo- 
cations. Although the blade section is constant from hub to tip, the increased pitch at the tip increases the loading of the tip section considerably.

Figure 12 compares calculated (top) and measured (bottom, Ref. 14) efficiency contours on a cross-channel surface located $1 / 3$ axial chord downstream of the trailing edge. The kinetic energy efficiency is theoretically independent of the axial position and is defined by the following:

$$
\eta=Q_{\text {actual }}^{2} / Q_{\text {ideal }}^{2}
$$

where

$$
Q_{\text {ideal }}^{2}=2 C_{p} T_{0}\left(1-\frac{T_{\text {actual }}}{T_{0}}\right)
$$

and $T_{0}$ is taken as constant.

The efficiency contours clearly delineate the endwall boundary layers and wake. The computed wake is thinner than the measured wake and has higher losses (lower efficiency) at the center. Integrating the efficiencies over the entire area gives a total efficiency of 0.960 for the real machine and 0.923 for the calculations. The discrepancy could be due to inadequate resolution of the thick, round trailing edge, an inadequate turbulence model, or possibly to unsteady vortex shedding in the real flow. The high calculated losses seem not to be due to numerical dissipation. Indeed, the computed wake appears to be less dissipative than the measured wake, and the computed loss is remarkably insensitive to the artificial viscosity coefficient. Considerable work is clearly needed in the area of modelling the flows leaving the blunt trailing edges commonly found on turbomachinery blades.

\section{CONCLUDING REMARKS}

A numerical analysis has been developed for threedimensional viscous internal flows. The analysis solves the 3-D Navier-Stokes equations written in a general body-fitted coordinate system, including rotation about the $x$-axis. The thin-layer approximation is made in the streamwise direction but all viscous terms are included in the cross-planes. The Baldwin-Lomax eddy-viscosity model is used for turbulent flows.

An explicit multistage Runge-Kutta scheme is used to solve the finite-difference form of the flow equations. A variable time step and implicit residual smoothing are used to accelerate the convergence of the scheme. Convergence rates are slow at low Mach numbers but reasonable for typical turbomachinery applications. We hope to improve convergence rates by adding multigrid to the code.

Results showing the development of a horseshoe vortex in a turbulent boundary layer ahead of a cylinder are presented to validate the analysis. Excellent agreement is found between the computed results and experimental data for this case.

Results for an annular turbine cascade also show horseshoe vortex development. Very good agreement was found between measured and computed surface pressure distributions. Computed losses were high and show the need for improved modelling of flows around blunt trailing edges commonly found in turbomachinery.
It is felt that with additional work and experience this analysis will prove to be a useful tool for investigating three-dimensional viscous flow phenomena in turbomachinery.

\section{REF ERENCES}

1. Chima, R. V., "Inviscid and Viscous Flows in Cascades with an Explicit Multiple-Grid Algorithm, AlA A Journal, Vol. 23, No. 10, Oct. 1985, pp. 1556-1563.

2. Chima, R. V., "Explicit Multigrid Algorithm for Quasi-Three-Dimensional Viscous Flows in Turbomachinery," J. Propulsion and Power, Vol. 3, No. 5, Sept. Oct. 1987, pp. 397-405.

3. Dawes, W. N., "A Numerical Analysis of the ThreeDimensional Viscous Flow in a Transonic Compressor Rotor and Comparison With Experiment," J. Turbomachinery, Vol. 109, Jan. 1987, pp. 83-90.

4. Subramanian, S. V., and Borzola, R., "Numerical Simulation of Three-Dimensional Flow Fields in Turbomachinery Blade Rows Using the Compressible Navier-Stokes Equations," AIAA Paper 871314, June, 1987.

5. Rai, M. M., "Unsteady Three-Dimensional NavierStokes Simulations of Turbine Rotor-Stator Interactions," AIA A Paper 87-2058, June, 1987.

6. Baldwin, B. S., and Lomax, H., "Thin-Layer Approximation and Algebraic Model for Separated Turbulent Flows, AIAA Paper 78-257, Jan. 1978.

7. Gessner, F. B., Po, J. K., “A Reynolds Stress Model for Turbulent Corner Flows - Part II: Comparisons Between Theory and Experiment, ${ }^{D}$ J. Fluids Engr., June, 1976, pp. 269-277.

8. Sorenson, R. L., “A Computer Program to Generate Two-Dimensional Grids About Airfoils and Other Shapes by the Use of Poisson's Equation," NASA TM-81198, 1980.

9. Jameson, A., Schmidt, W., and Turkel, E., “Numerical Solutions of the Euler Equations by Finite Volume Methods Using Runge-Kutta Time-Stepping Schemes," AIAA Paper 81-1259, June 1981.

10. Hollanders, H., Lerat, A., and Peyret, R., "ThreeDimensional Calculation of Transonic Viscous Flows by an Implicit Method," AIAA Journal, Vol. 23, No. 11, Nov. 1985, pp. 1670-1678.

11. Jameson, A., and Baker, T. J., "Solution of the Euler Equations for Complex Configurations," AIAA Paper 83-1929, July 1983.

12. Eckerle, W. A., and Langston, L. S., "Measurements of a Turbulent Horseshoe Vortex Formed Around a Cylinder," NASA Contractor Report 3986, June, 1986.

13. Goldman, L. J., and Seasholtz, R. G., "Laser Anemometer Measurements in an Annular Cascade of Core Turbine Vanes and Comparison With Theory," NASA Technical Paper 2018, June, 1982.

14. Goldman, L. J., and McLallin, K. L., "Cold-Air Annular-Cascade Investigation of Aerodynamic Performance of Core-Engine-Cooled Turbine Vanes. I: Solid-Vane Performance and Facility Description," NASA TM X-3224, 1975. 


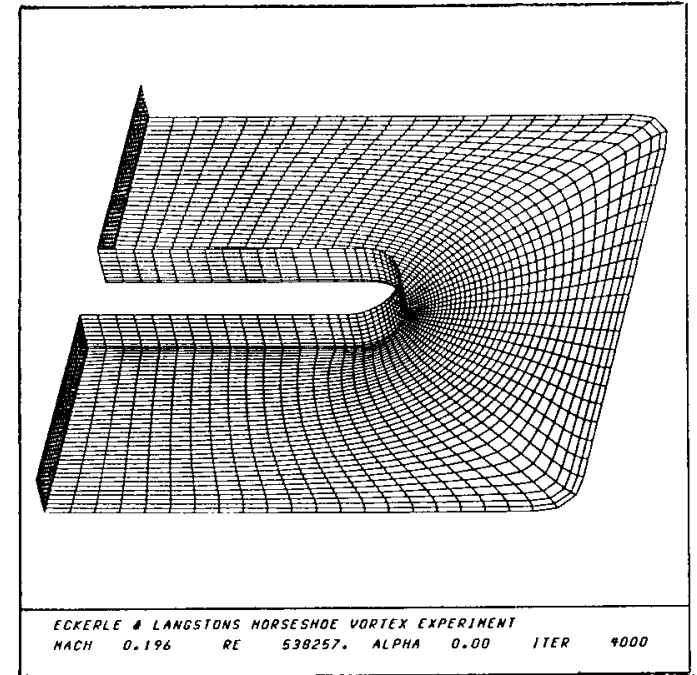

Figure 1. Computational grid for the horseshoe vortex problem.

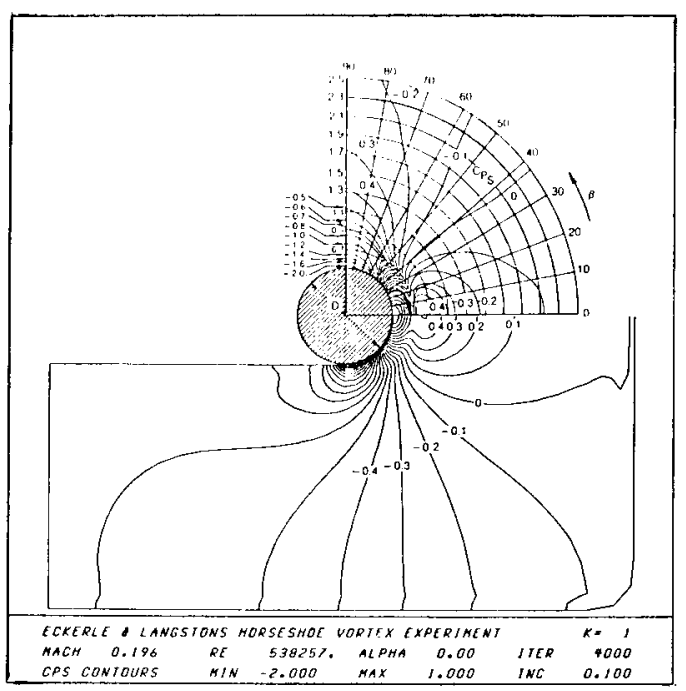

Figure 3. Comparison of measured (top) and computed (bottom) static pressure contours on the endwall.

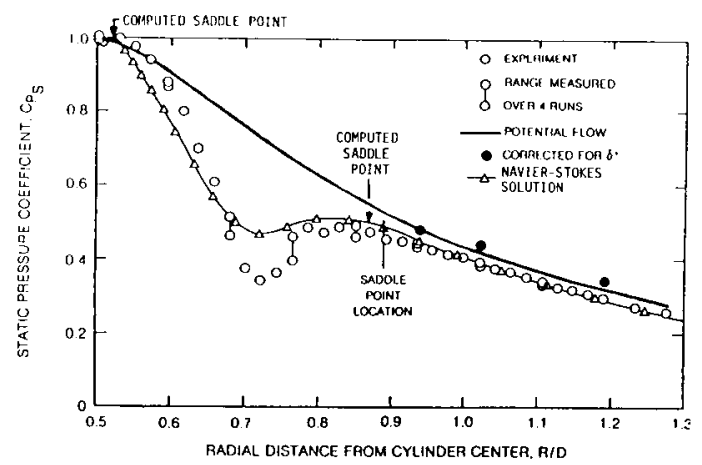

Figure 5. Comparison of measured and computed static pressure distributions on the endwall symmetry line.

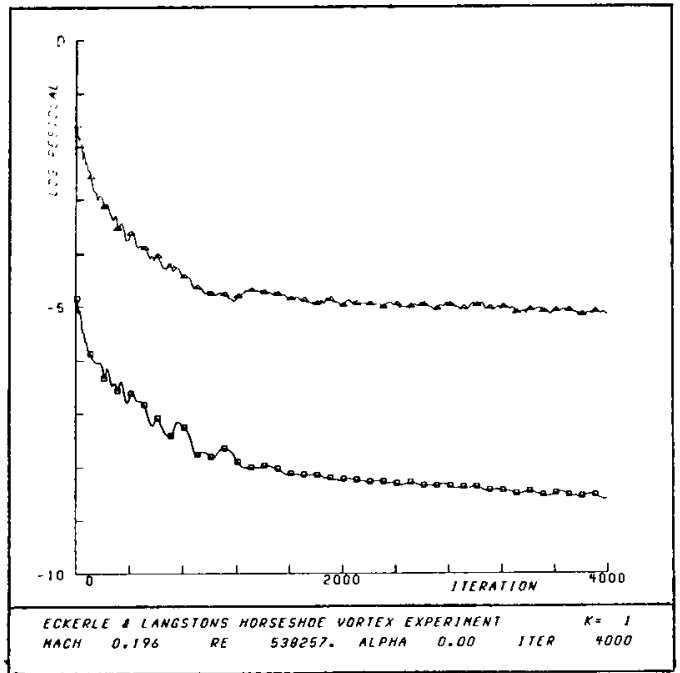

Figure 2. Convergence history for the horseshoe vortex problem.

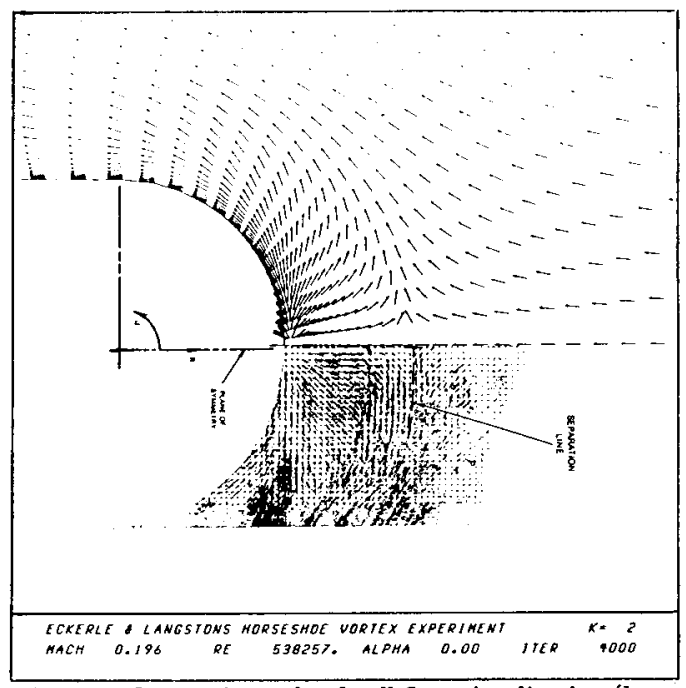

Figure 4. Comparison of endwall flow visualization (bottom) and computed flow direction vectors (top) ahead of the cylinder.

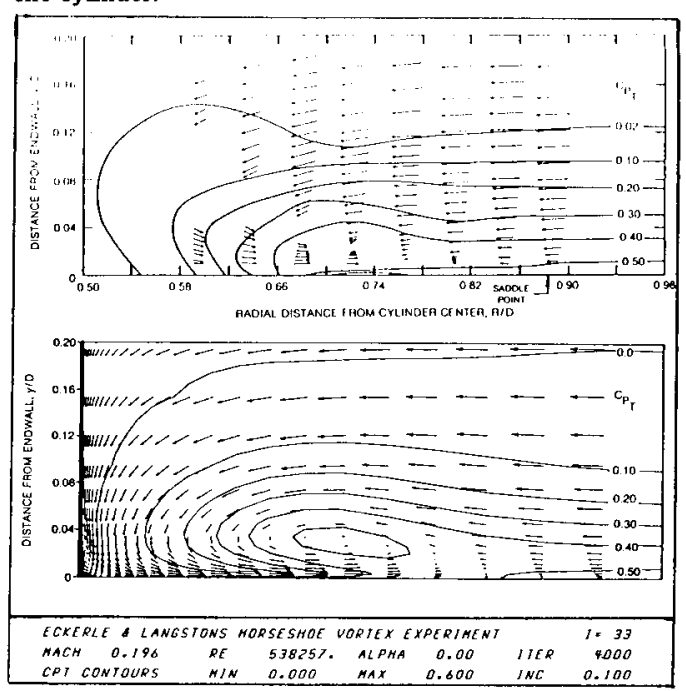

Figure 6. Comparison of measured (top) and computed (bottom) velocity vectors and total pressure loss contours on the symmetry plane. 


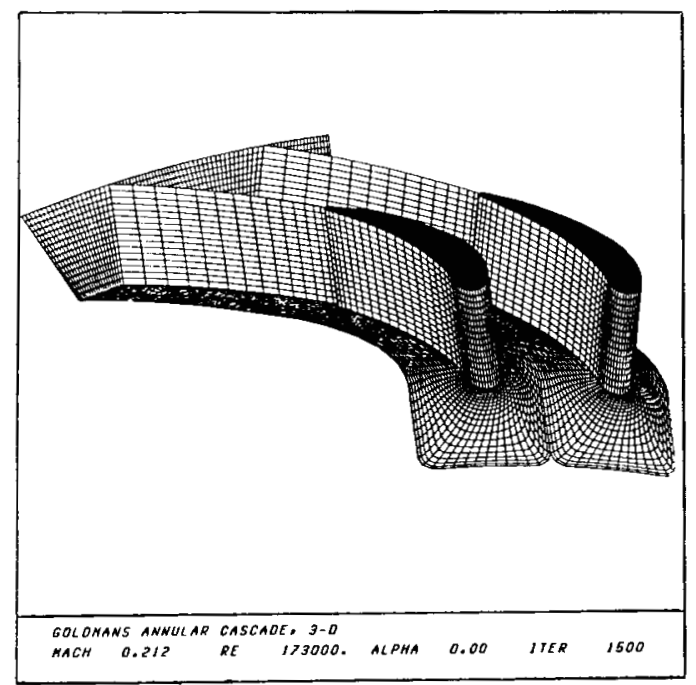

Figure 7. Computational grid for the annular turbine cascade.

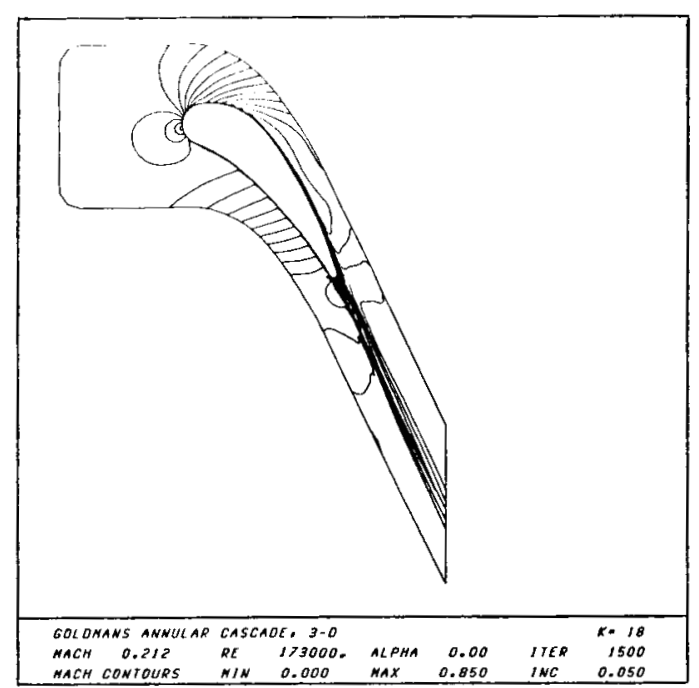

Figure 9. Mach number contours at mid-span.

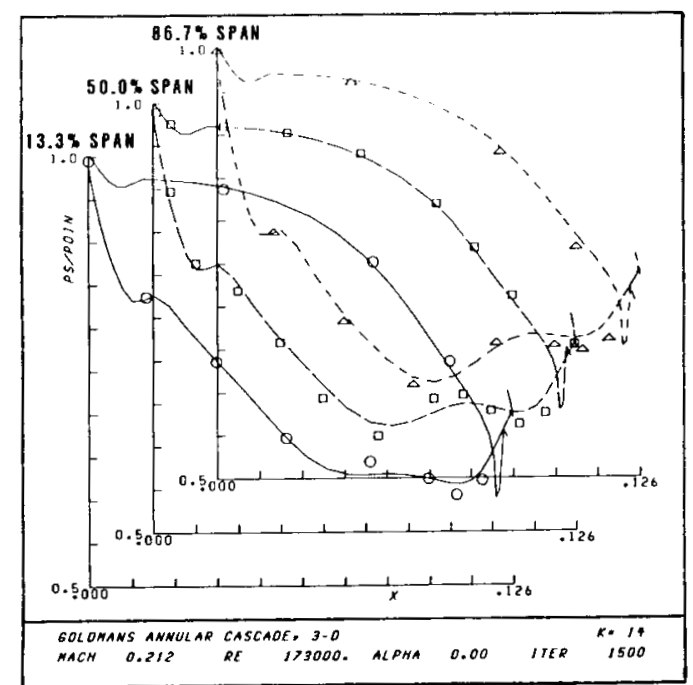

Figure 11. Comparison of measured and computed pressure distributions at three span-wise locations.

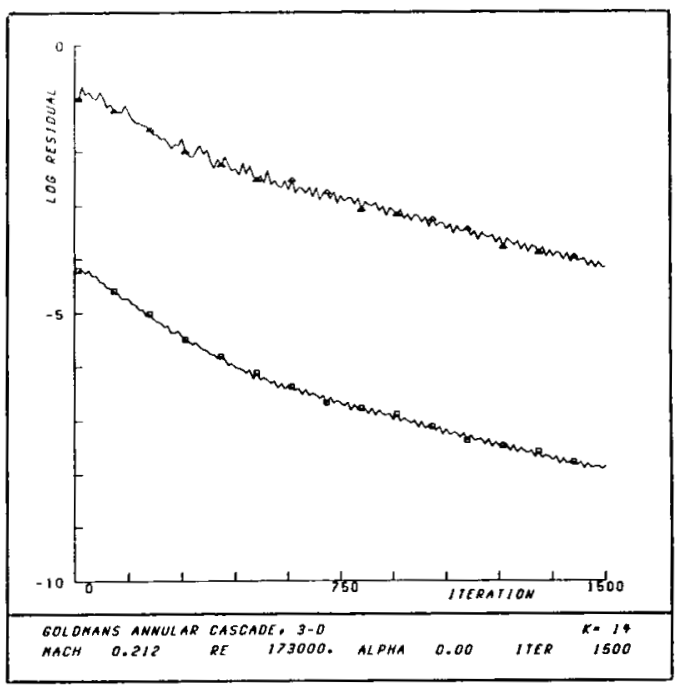

Figure 8. Convergence history for the annular turbine cascade problem.

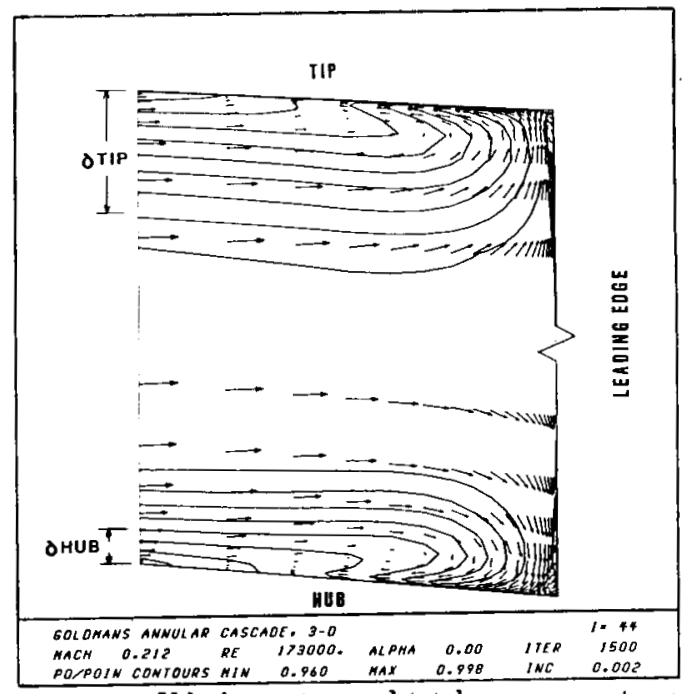

Figure 10. Velocity vectors and total pressure contours in the endwall boundary layers near the leading edge.

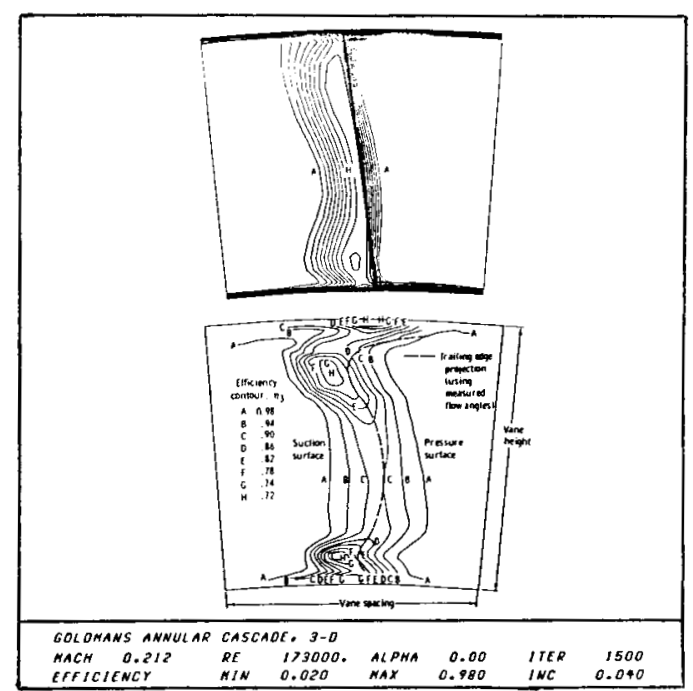

Figure 12. Comparison of measured (bottom) and computed (top) efficiency contours $1 / 3$ axial chord downstream of the trailing edge. 


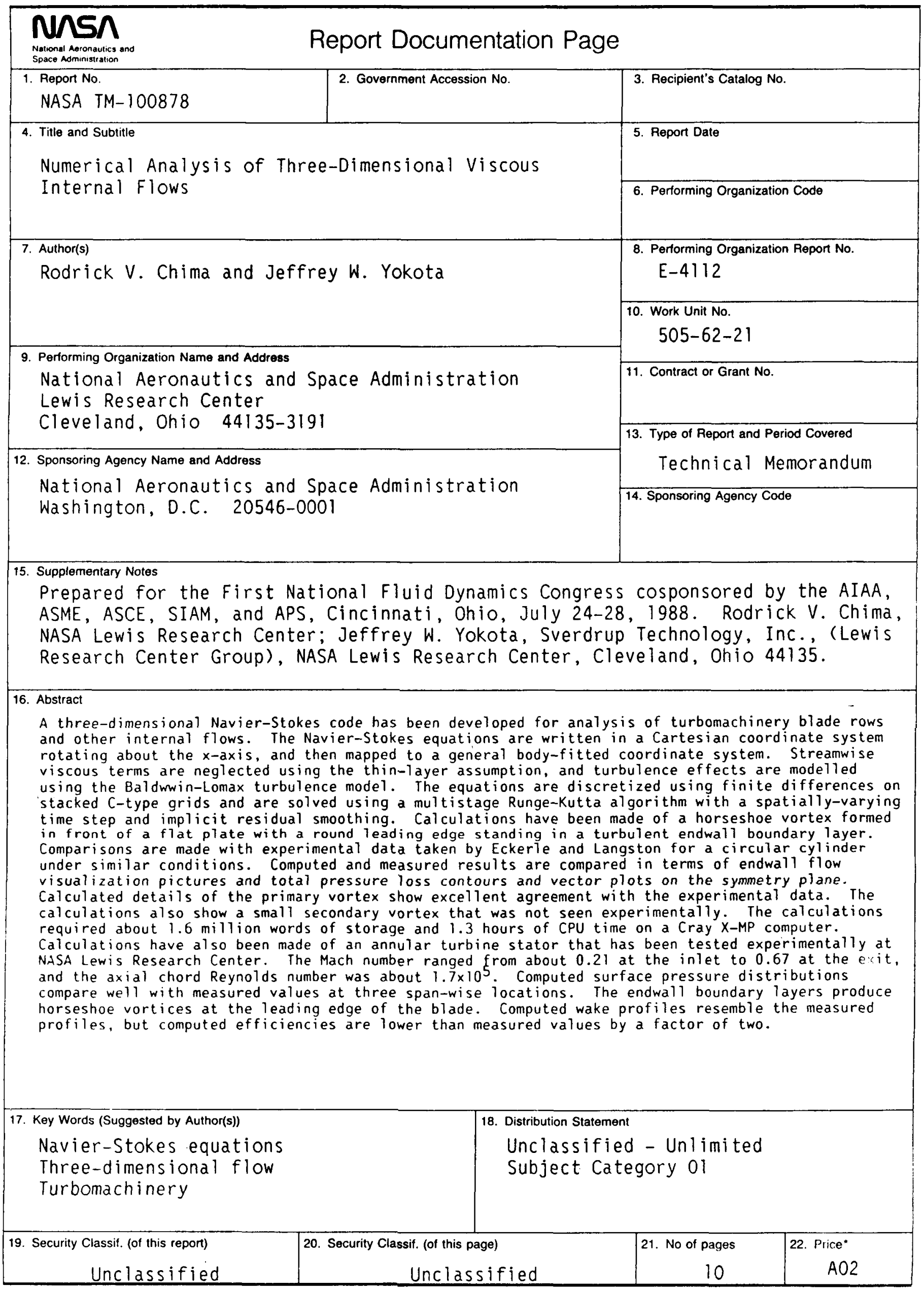

16. Abstract
A three-d
and other
rotating
viscous te
using the
stacked C-
time step
in front
Comparisons
under sim
visual izat
Calculated
calculation
required
Calculations
NasA Lewis
and the ax
compare we
horseshoe
profiles, 